\title{
ORDER STATISTICS WITH DISCRETE DISTRIBUTIONS AND PROBABILITY THAT TIES OCCUR SOMEWHERE
}

\author{
Masayuki Uchida*
}

\begin{abstract}
Let $X_{1: n} \leq X_{2: n} \leq \cdots \leq X_{n: n}$ denote the order statistics of a random sample of size $n$ with an arbitrary distribution. First, we obtain general expressions of the joint distribution of $k$ order statistics represented by $X_{i_{1}: n}, X_{i_{2}: n}, \ldots, X_{i_{k}: n}$, where $1 \leq i_{1}<i_{2}<\cdots<i_{k} \leq n$. From these expressions, the joint distribution of $k$ order statistics with a discrete distribution can be obtained exactly by means of computer algebra. We also obtain the probability that ties occur somewhere when the distribution is discrete. Finally with attention to bootstrap sample having a finite population, we obtain some examples for a bootstrap sample by using our results.
\end{abstract}

\section{Introduction}

Let $X$ be a random variable (rv) having a cumulative distribution function (cdf) $F(x)$. Let the probability mass function (pmf) be $m(x)=F(x)-F(x-)$. Let $S$ be the set of all points $x$ at which the pmf $m(x)$ is positive, that is, $S=\{x: m(x)>0\}$. Of course, $S$ is a countable set. When the $\operatorname{cdf} F(x)$ of $X$ is increasing only in jumps, we say that the distribution of $X$ is discrete. In other words, when the cdf $F(x)$ of $X$ is completely determined by jump points $x_{1}, x_{2}, \ldots$ and their pmf $m\left(x_{1}\right), m\left(x_{2}\right), \ldots$, the distribution of $X$ is discrete. Let $X_{1: n} \leq X_{2: n} \leq \cdots \leq X_{n: n}$ denote the order statistics of a random sample of size $n$ with the distribution of $X$. When the parent distribution is continuous, extensive work on order statistics has been done ( see, e.g., David (1981) ). Recently for a discrete parent, several papers on order statistics have appeared. Arnold, Balakrishnan and Nagaraja (1992) have considered some distributional properties of discrete order statistics.

There were some expressions for the joint distribution of $k$ order statistics represented by $X_{i_{1}: n}, X_{i_{2}: n}, \ldots, X_{i_{k}: n}$, where $1 \leq i_{1}<i_{2}<\cdots<i_{k} \leq n$. Abdel-Aty (1954) was the first to derive the joint pmf of discrete order statistics for $k=2$. Khatri (1962) derived the joint pmf of discrete order statistics in an integral form for $k \leq 3$, but proved it for only $k \leq 2$ for the case of no ties. Nagaraja (1992) obtained a general expression for the joint pmf of $k$ discrete order statistics in the integral form of the joint probability density function (pdf) of $k$ order statistics with uniform distribution on $(0,1)$. Reiss (1989) obtained the joint cdf of $k$ order statistics with an arbitrary cdf $F(x)$.

In Section 2, we obtain more general expressions for the joint distribution of $k$ order statistics. From these expressions we can obtain the joint distribution of $k$ order statistics exactly by means of computer algebra. It is well known that, in the continuous case, the probability that two consecutive order statistics are equal (i.e., probability of tie ) is zero.

*Department of Mathematical Science, Faculty of Engineering Science, Osaka University

Key words: Order statistics, Discrete distribution, Probability of tie, Bootstrap sample. 
But it is not true when the distribution is discrete. Gupta and Gupta (1981) obtained the probability of ties which is $P\left(X_{i: n}=X_{j: n}=\alpha\right)$ for $i \neq j$.

In Section 3, we obtain the probability that at least two consecutive order statistics are equal, that is, the probability that ties occur somewhere.

In the last section, with attention to bootstrap sample which is a random sample from a finite population, we apply our results to bootstrap sample. For example, we obtain the exact distribution of interquartile range of bootstrap sample by using our results.

\section{The joint distribution of $k$ order statistics}

Let $X_{1}, X_{2}, \ldots, X_{n}$ be independent and identically distributed (i.i.d.) rvs with common cdf $F(x)$. Let the quantile function (qf) of a $\operatorname{cdf} F(x)$ be $G(t)=\inf \{x: F(x) \geq t\}$ for $0<t<1$. Let $U$ be a uniform rv distributed over $(0,1)$. Let $U_{1}, U_{2}, \ldots, U_{n}$ be a random sample from the distribution of $U$ and let $U_{1: n}<U_{2: n}<\cdots<U_{n: n}$ be the set of the order statistics. In this section, we consider the joint distribution of $k$ order statistics represented by $X_{i_{1}: n}, X_{i_{2}: n}, \ldots, X_{i_{k}: n}$, where $1 \leq i_{1}<i_{2}<\cdots<i_{k} \leq n$.

We now introduce some equations which are used extensively in proving the main results.

For any cdf $F(x)$ and its qf $\mathrm{G}(\mathrm{t})$, we define $\xi(\omega) \equiv G(U(\omega))$. Then we obtain the following equations,

$$
\begin{gathered}
\{\xi(\omega) \leq x\}=\{U(\omega) \leq F(x)\}, \\
\left\{x_{1}<\xi(\omega) \leq x_{2}\right\}=\left\{F\left(x_{1}\right)<U(\omega) \leq F\left(x_{2}\right)\right\} .
\end{gathered}
$$

The proof is in Shorack and Wellner (1986 p. 4).

For any cdf $F(x)$ and its qf $G(t)$, we define $\xi_{i}(\omega) \equiv G\left(U_{i}(\omega)\right)$ for $i=1,2, \ldots, n$. Then we obtain $\xi_{1}, \xi_{2}, \ldots, \xi_{n}$ which are i.i.d. rvs with common cdf $F(x)$ and

$$
\left(\xi_{1: n}, \xi_{2: n}, \ldots, \xi_{n: n}\right)=\left(G\left(U_{1: n}\right), G\left(U_{2: n}\right), \ldots, G\left(U_{n: n}\right)\right) .
$$

The proof is in Reiss (1989 p. 17).

We obtain a general expression for the joint distribution of $k$ order statistics. It is given by the following result.

\section{Thorem 2.1.}

For $1 \leq i_{1}<i_{2}<\cdots<i_{k} \leq n$, the joint distribution of $X_{i_{1}: n}, X_{i_{2}: n}, \ldots, X_{i_{k}: n}$ is given by

$$
\begin{aligned}
& P\left(X_{i_{1}: n} \in B_{i_{1}}, X_{i_{2}: n} \in B_{i_{2}}, \ldots, X_{i_{k}: n} \in B_{i_{k}}\right) \\
& =P\left(U_{i_{1}: n} \in G^{-1}\left(B_{i_{1}}\right), U_{i_{2}: n} \in G^{-1}\left(B_{i_{2}}\right), \ldots, U_{i_{k}: n} \in G^{-1}\left(B_{i_{k}}\right)\right),
\end{aligned}
$$

where $B_{i_{r}}(r=1,2, \ldots, k)$ are Borel sets

and $G^{-1}$ is the inverse image of $\mathrm{G}$, that is,$G^{-1}\left(B_{i_{k}}\right)=\left\{x \mid x \in(0,1), G(x) \in B_{i_{k}}\right\}$.

Proof. From the equation (3), we obtain

$$
\left(\xi_{i_{1}: n}, \xi_{i_{2}: n}, \ldots, \xi_{i_{k}: n}\right)=\left(G\left(U_{i_{1}: n}\right), G\left(U_{i_{2}: n}\right), \ldots, G\left(U_{i_{k}: n}\right)\right)
$$

Since $X_{1}, X_{2}, \ldots, X_{n}$ are i.i.d. rvs with common cdf $F(x)$, we have

$$
\left(X_{i_{1}: n}, X_{i_{2}: n}, \ldots, X_{i_{k}: n}\right) \stackrel{d}{=}\left(G\left(U_{i_{1}: n}\right), G\left(U_{i_{2}: n}\right), \ldots, G\left(U_{i_{k}: n}\right)\right)
$$


where $\stackrel{d}{=}$ stands for equality in distribution.

Then we obtain

$$
\begin{aligned}
& P\left(X_{i_{1}: n} \in B_{i_{1}}, X_{i_{2}: n} \in B_{i_{2}}, \ldots, X_{i_{k}: n} \in B_{i_{k}}\right) \\
& =P\left(G\left(U_{i_{1}: n}\right) \in B_{i_{1}}, G\left(U_{i_{2}: n}\right) \in B_{i_{2}}, \ldots, G\left(U_{i_{k}: n}\right) \in B_{i_{k}}\right) \\
& =P\left(U_{i_{1}: n} \in G^{-1}\left(B_{i_{1}}\right), U_{i_{2}: n} \in G^{-1}\left(B_{i_{2}}\right), \ldots, U_{i_{k}: n} \in G^{-1}\left(B_{i_{k}}\right)\right) .
\end{aligned}
$$

This completes the proof.

Remark 1. This result holds for any cdf $F(x)$, that is, even if $F(x)$ is not absolutely continuous w.r.t. Lebesgue measure, the joint distribution of $X_{i_{1}: n}, X_{i_{2}: n}, \ldots, X_{i_{k}: n}$ is given by the joint distribution of $U_{i_{1}: n}, U_{i_{2}: n}, \ldots, U_{i_{k}: n}$.

\section{Corollary 2.1.}

For $1 \leq i_{1}<i_{2}<\cdots<i_{k} \leq n$, the joint cdf of $X_{i_{1}: n}, X_{i_{2}: n}, \ldots, X_{i_{k}: n}$ is given by

$$
\begin{aligned}
& P\left(X_{i_{1}: n} \leq x_{i_{1}}, X_{i_{2}: n} \leq x_{i_{2}}, \ldots, X_{i_{k}: n} \leq x_{i_{k}}\right) \\
& =\int_{0}^{F\left(x_{i_{1}}\right)} \int_{0}^{F\left(x_{i_{2}}\right)} \cdots \int_{0}^{F\left(x_{i_{k}}\right)} f_{i_{1}, i_{2}, \ldots, i_{k}}\left(u_{i_{1}}, u_{i_{2}}, \ldots, u_{i_{k}}\right) d u_{i_{1}} d u_{i_{2}} \cdots d u_{i_{k}},
\end{aligned}
$$

where $f_{i_{1}, i_{2}, \ldots, i_{k}}\left(u_{i_{1}}, u_{i_{2}}, \ldots, u_{i_{k}}\right)$ is the joint pdf of $U_{i_{1}: n}, U_{i_{2}: n}, \ldots, U_{i_{k}: n}$, that is, for $0<u_{i_{1}}<u_{i_{2}}<\cdots<u_{i_{k}}<1$,

$$
\begin{aligned}
& f_{i_{1}, i_{2}, \ldots, i_{k}}\left(u_{i_{1}}, u_{i_{2}}, \ldots, u_{i_{k}}\right) \\
& =\frac{n !}{\left(i_{1}-1\right) !\left(i_{2}-i_{1}-1\right) ! \cdots\left(n-i_{k}\right) !} u_{i_{1}}^{i_{1}-1}\left(u_{i_{2}}-u_{i_{1}}\right)^{i_{2}-i_{1}-1} \cdots\left(1-u_{i_{k}}\right)^{n-i_{k}} .
\end{aligned}
$$

Proof. By setting $B_{i}=\left(-\infty, x_{i}\right]$ in Theorem 2.1 and using the equation (1), we obtain the result.

This result agrees with the Corollary 1.2.7 given by Reiss (1989 p. 18).

\section{Corollary 2.2.}

For $1 \leq i_{1}<i_{2}<\cdots<i_{k} \leq n$, the joint pmf of $X_{i_{1}: n}, X_{i_{2}: n}, \ldots, X_{i_{k}: n}$ is given by

$$
\begin{aligned}
& P\left(X_{i_{1}: n}=x_{i_{1}}, X_{i_{2}: n}=x_{i_{2}}, \ldots, X_{i_{k}: n}=x_{i_{k}}\right) \\
& =\int_{F\left(x_{i_{1}}-\right)}^{F\left(x_{i_{1}}\right)} \int_{F\left(x_{i_{2}}-\right)}^{F\left(x_{i_{2}}\right)} \cdots \int_{F\left(x_{i_{k}}-\right)}^{F\left(x_{i_{k}}\right)} f_{i_{1}, i_{2}, \ldots, i_{k}}\left(u_{i_{1}}, u_{i_{2}}, \ldots, u_{i_{k}}\right) d u_{i_{1}} d u_{i_{2}} \cdots d u_{i_{k}},
\end{aligned}
$$

where $f_{i_{1}, i_{2}, \ldots, i_{k}}\left(u_{i_{1}}, u_{i_{2}}, \ldots, u_{i_{k}}\right)$ is the joint pdf of $U_{i_{1}: n}, U_{i_{2}: n}, \ldots, U_{i_{k}: n}$.

Proof. By setting $B_{i}=\left\{x_{i}\right\}$ in Theorem 2.1 and using the equation (2), we obtain the result.

This result agrees with the formula given by Nagaraja (1992).

Remark 2. Of course, Corollaries 2.1 and 2.2.hold for any cdf $F(x)$. These results mean that the joint distribution of $X_{i_{1}: n}, X_{i_{2}: n}, \ldots, X_{i_{k}: n}$ is represented in the integral form of the joint pdf of $U_{i_{1}: n}, U_{i_{2}: n}, \ldots, U_{i_{k}: n}$. The joint pdf of $k$ order statistics with the uniform distribution on $(0,1)$ is expressed as a polynomial in $k$ variables, $u_{i_{1}}, u_{i_{2}}, \ldots, u_{i_{k}}$, that is, $(2.1)$ is the polynomial expression. This means that the joint distribution of $X_{i_{1}: n}, X_{i_{2}: n}, \ldots, X_{i_{k}: n}$ is 
represented in the integral form of the polynomial. By these results, we can easily obtain the joint distribution of $k$ order statistics by means of computer algebra. Here we use a computer algebra system, REDUCE (see Hearn(1984)) in order to obtain the joint distribution of $k$ order statistics. For example, the expression for the joint pmf of 2 order statistics of a random sample of size 7 with a cdf $F(x)$ is easily obtained by means of the REDUCE system. Of course, Corollaries 2.1 and 2.2 are expressed as an integral form of a polynomial. But, what we want to emphasize here is its feasibility in our usual computer system. Certainly the joint distribution of $X_{i_{1}: n}, X_{i_{2}: n}, \ldots, X_{i_{k}: n}$ is also represented by means of enumerative combinatrics. But the representation is not better for computational purposes, especially for discrete case. It is notable that the joint pmf of $k$ order statistics can be obtained exactly by computers when we use Corollary 2.2 and computer algebra system like REDUCE which can execute integral arithmetic of polynomials. See Appendix 1.

\section{Probability that ties occur somewhere}

Let $X$ be a discrete rv having cdf $F(x)$ and pmf $m(x)=F(x)-F(x-)$ and $S=\{x$ : $m(x)>0\}$. Let $X_{1: n} \leq X_{2: n} \leq \cdots \leq X_{n: n}$ denote the order statistics of a random sample of size $n$ with the distribution of $X$. In this section, we obtain the probability that ties occur somewhere, that is, at least two consecutive order statistics are equal. It is given by the following result.

\section{Theorem 3.1.}

Let $A$ be the event that ties occur somewhere. Then we obtain

$$
P(A)=1-n ! \sum^{\prime} \prod_{i=1}^{n} m\left(x_{i}\right),
$$

where the summation $\sum^{\prime}$ is over all $x_{1}, x_{2}, \ldots, x_{n} \in S$ such that $x_{1}<x_{2}<\cdots<x_{n}$. Proof. Let $B$ be the event that no ties occur. Then we obtain

$$
\begin{aligned}
P(B) & =\sum^{\prime} P\left(X_{1: n}=x_{1}, X_{2: n}=x_{2}, \ldots, X_{n: n}=x_{n}\right) \\
& =\sum^{\prime} n ! \prod_{i=1}^{n} m\left(x_{i}\right),
\end{aligned}
$$

where the summation $\sum^{\prime}$ is over all $x_{1}, x_{2}, \ldots, x_{n} \in S$ such that $x_{1}<x_{2}<\cdots<x_{n}$.

$$
P(A)=1-P(B)=1-\sum^{\prime} n ! \prod_{i=1}^{n} m\left(x_{i}\right) .
$$

This completes the proof.

Remark 3. Let \#S be the number of elements of the set $S$.

(i) If $\# S<n$, then there does not exist the summation $\Sigma^{\prime}$ that is over all $x_{1}, x_{2}, \ldots, x_{n} \in S$ such that $x_{1}<x_{2}<\cdots<x_{n}$.

This means

$$
\sum^{\prime} n ! \prod_{i=1}^{n} m\left(x_{i}\right)=0
$$


that is,

$$
P(A)=1 \text {. }
$$

(ii) If \# $S=n$, then there exists only one summation $\sum^{\prime}$ that is over all $x_{1}, x_{2}, \ldots, x_{n} \in S$ such that $x_{1}<x_{2}<\cdots<x_{n}$.

This means

$$
\begin{aligned}
P(B) & =P\left(X_{1: n}=x_{1}, X_{2: n}=x_{2}, \ldots, X_{n: n}=x_{n}\right) \\
& =n ! \prod_{i=1}^{n} m\left(x_{i}\right),
\end{aligned}
$$

that is,

$$
P(A)=1-n ! \prod_{i=1}^{n} m\left(x_{i}\right)
$$

\section{Application to bootstrap sample}

Let $X_{1}, X_{2}, \ldots, X_{n}$ be a random sample of size $n$ with a continuous cdf $F(x)$ and let $X_{1: n}<X_{2: n}<\cdots<X_{n: n}$ be the set of order statistics. $F_{n}(x)$ denotes the empirical distribution function of the variables $X_{1}, X_{2}, \ldots, X_{n}$. Let $X_{1}^{*}, X_{2}^{*}, \ldots, X_{k}^{*}$ be a random sample of size $k$ with the cdf $F_{n}(x)$ and let $X_{1: k}^{*} \leq X_{2: k}^{*} \leq \cdots \leq X_{k: k}^{*}$ be the set of order statistics $(k \leq n)$. In other words, we define $X_{i: n}(\omega)=x_{i}$ for $i=1,2, \ldots, n$, then $X_{1}^{*}, X_{2}^{*}, \ldots, X_{k}^{*}$ are a bootstrap sample from the finite population $S^{*}=\left\{x_{1}, x_{2}, \ldots, x_{n}\right\}$ with the discrete cdf $F_{n}(x)$ and the pmf $f_{n}(x)=F_{n}(x)-F_{n}(x-)=1 / n$. In this section, we apply our results to some examples for a bootstrap sample.

Example 4.1. (Exact distribution of median of bootstrap sample)

We assume an odd sample size $k=2 m-1$ for convenience. By using Corollary 2.1, we obtain

$$
\begin{aligned}
P^{*}\left(X_{m: k}^{*} \leq x\right) & =P^{*}\left(U_{m: k} \leq F_{n}(x)\right) \\
& =\int_{0}^{F_{n}(x)} \frac{k !}{(m-1) !(k-m) !} u^{m-1}(1-u)^{k-m} d u .
\end{aligned}
$$

This result agrees with the one given by Efron (1979) and Beran and Ducharme (1991).

Example 4.2. (Exact distribution of interquartile range of bootstrap sample)

We assume a sample size $k=4 m-1$ for convenience. By using Corollary 2.2, we obtain

$$
\begin{aligned}
P^{*}\left(X_{3 m: k}^{*}-X_{m: k}^{*} \leq x\right) & =\sum^{\prime} P^{*}\left(X_{m: k}^{*}=y_{m}, X_{3 m: k}^{*}=y_{3 m}\right) \\
& =\sum^{\prime} \int_{F_{n}\left(y_{m}-\right)}^{F_{n}\left(y_{m}\right)} \int_{F_{n}\left(y_{3 m}-\right)}^{F_{n}\left(y_{3 m}\right)} f_{m, 3 m}\left(u_{m}, u_{3 m}\right) d u_{m} d u_{3 m},
\end{aligned}
$$

where the summation $\sum^{\prime}$ is over all $y_{m}, y_{3 m} \in S^{*}$ such that $y_{m} \leq y_{3 m}, y_{3 m}-y_{m} \leq x$ and $f_{m, 3 m}\left(u_{m}, u_{3 m}\right)$ is the joint pdf of $U_{m: k}, U_{3 m: k}$. 
Remark 4. By using Example 4.2 and the REDUCE system, we can immediately obtain the exact distribution of interquartile range of bootstrap sample. See Appendix 2.

Example 4.3. (Probability that ties occur somewhere in bootstrap sample)

Let $A_{k, n}^{*}$ be the event that ties occur somewhere in bootstrap sample of size $k$ with the cdf $F_{n}(x)$.

From Theorem 3.1, we obtain

$$
\begin{aligned}
P^{*}\left(A_{k, n}^{*}\right) & =1-k ! \sum^{\prime} \prod_{i=1}^{k} f_{n}\left(x_{i}\right) \\
& =1-k ! \sum^{\prime} \prod_{i=1}^{k} \frac{1}{n} \\
& =1-k ! \sum^{\prime}\left(\frac{1}{n}\right)^{k}
\end{aligned}
$$

where the summation $\sum^{\prime}$ is over all $x_{1}, x_{2}, \ldots, x_{k} \in S^{*}$ such that $x_{1}<x_{2}<\cdots<x_{k}$. Then we obtain

$$
\begin{aligned}
P^{*}\left(A_{k, n}^{*}\right) & =1-k !\left(\begin{array}{l}
n \\
k
\end{array}\right)\left(\frac{1}{n}\right)^{k} \\
& =1-\left(1-\frac{1}{n}\right)\left(1-\frac{2}{n}\right) \cdots\left(1-\frac{k-1}{n}\right) .
\end{aligned}
$$

\section{Appendix 1.}

Let $X$ denote a nonnegative integer valued rv having a cdf $F(x)$. Let $X_{1: n} \leq X_{2: n} \leq$ $\cdots \leq X_{n: n}$ denote the order statistics of a random sample of size $n$ with the distribution of $X$. By using Corollary 2.2, we obtain the following expression for the joint pmf of $k$ order statistics with the cdf $F(x)$, that is, for $1 \leq i_{1}<i_{2}<\cdots<i_{k} \leq n$,

$$
\begin{aligned}
& P\left(X_{i_{1}: n}=x_{i_{1}}, X_{i_{2}: n}=x_{i_{2}}, \ldots, X_{i_{k}: n}=x_{i_{k}}\right) \\
& =\int_{F\left(x_{i_{1}}-1\right)}^{F\left(x_{i_{1}}\right)} \int_{F\left(x_{i_{2}}-1\right)}^{F\left(x_{i_{2}}\right)} \cdots \int_{F\left(x_{i_{k}}-1\right)}^{F\left(x_{i_{k}}\right)} f_{i_{1}, i_{2}, \ldots, i_{k}}\left(u_{i_{1}}, u_{i_{2}}, \ldots, u_{i_{k}}\right) d u_{i_{1}} d u_{i_{2}} \cdots d u_{i_{k}},
\end{aligned}
$$

where $x_{i_{1}}, x_{i_{2}}, \ldots, x_{i_{k}}$ are integers and

$f_{i_{1}, i_{2}, \ldots, i_{k}}\left(u_{i_{1}}, u_{i_{2}}, \ldots, u_{i_{k}}\right)$ is the joint $\mathrm{pdf}$ of $U_{i_{1}: n}, U_{i_{2}: n}, \ldots, U_{i_{k}: n}$.

We can obtain the above expression in the polynomial form of the cdf $F(x)$. In other words, the joint pmf of $k$ order statistics with the discrete $\operatorname{cdf} F(x)$ can be expressed as the polynomial form of the cdf $F(x)$.

Now we give the corresponding programs available in the REDUCE system.

OPERATOR I,X,F,FAC,C,G,U;

PROCEDURE JPMF $(\mathrm{N}, \mathrm{K}, \mathrm{II}, \mathrm{XX}, \mathrm{F})$;

BEGIN SCALAR Y;

FOR J:=1:K DO I(J):=PART(II,J); 
FOR J:=1:K DO X(J):=PART $(\mathrm{XX}, \mathrm{J})$;

$\mathrm{I}(0):=0 ; \mathrm{I}(\mathrm{K}+1):=\mathrm{N}+1 ; \mathrm{U}(0):=0 ; \mathrm{U}(\mathrm{K}+1):=1$;

FOR ALL J LET FAC (J)=IF J=0 THEN 1 ELSE FOR K2:=1:J PRODUCT K2;

$\mathrm{C}(\mathrm{K}):=$ FOR $\mathrm{R}:=1: \mathrm{K}+1$ PRODUCT FAC $(\mathrm{I}(\mathrm{R})-\mathrm{I}(\mathrm{R}-1)-1)$;

$\mathrm{G}(\mathrm{K}):=$ FOR R: $=1: \mathrm{K}+1$ PRODUCT $(\mathrm{U}(\mathrm{R})-\mathrm{U}(\mathrm{R}-1))^{* *}(\mathrm{I}(\mathrm{R})-\mathrm{I}(\mathrm{R}-1)-1)$;

$\mathrm{Y}:=\mathrm{FAC}(\mathrm{N}) / \mathrm{C}(\mathrm{K}) * \mathrm{G}(\mathrm{K})$;

$\mathrm{R}:=1$;

WHILE $\mathrm{R}<\mathrm{K}$ DO $<<\mathrm{Y}:=\mathrm{INT}(\mathrm{Y}, \mathrm{U}(\mathrm{R}))$;IF $\mathrm{X}(\mathrm{R}+1)-\mathrm{X}(\mathrm{R})=0$ THEN

$\mathrm{Y}:=\mathrm{SUB}(\mathrm{U}(\mathrm{R})=\mathrm{U}(\mathrm{R}+1), \mathrm{Y})-\mathrm{SUB}(\mathrm{U}(\mathrm{R})=\mathrm{F}(\mathrm{X}(\mathrm{R})-1), \mathrm{Y})$ ELSE

$\mathrm{Y}:=\mathrm{SUB}(\mathrm{U}(\mathrm{R})=\mathrm{F}(\mathrm{X}(\mathrm{R})), \mathrm{Y})-\mathrm{SUB}(\mathrm{U}(\mathrm{R})=\mathrm{F}(\mathrm{X}(\mathrm{R})-1), \mathrm{Y})$;

$\mathrm{R}:=\mathrm{R}+1>>$;

$\mathrm{Y}:=\mathrm{INT}(\mathrm{Y}, \mathrm{U}(\mathrm{K}))$;

$\mathrm{Y}:=\mathrm{SUB}(\mathrm{U}(\mathrm{K})=\mathrm{F}(\mathrm{X}(\mathrm{K})), \mathrm{Y})-\mathrm{SUB}(\mathrm{U}(\mathrm{K})=\mathrm{F}(\mathrm{X}(\mathrm{K})-1), \mathrm{Y})$;

RETURN Y;

END;

END;

By using the above programs, we can immediately give formulas of the joint pmf of $k$ order statistics with the cdf $F(x)$. Here we obtain the joint pmf of 2 order statistics of a random sample of size 7 with a cdf $F(x)$. Let $X_{1}, X_{2}, \ldots, X_{7}$ be a random sample of size 7 with a cdf $F(x)$ and let $X_{1: 7} \leq X_{2: 7} \leq \cdots \leq X_{7: 7}$ denote the order statistics of this sample. We consider the joint pdf of 2 order statistics represented by $X_{2: 7}$ and $X_{6: 7}$. By using Corollary 2.2 and the REDUCE system, we can obtain the following two equations.

For integers $x 1$ and $x 2$ with $x 1 \neq x 2$,

$$
\begin{aligned}
P( & \left.X_{2: 7}=x 1, X_{6: 7}=x 2\right)=\int_{F(x 1-1)}^{F(x 1)} \int_{F(x 2-1)}^{F(x 2)} f_{2,6}\left(u_{2}, u_{6}\right) d u_{2} d u_{6} \\
= & 7\left(12 F(x 1-1)^{5} F(x 2-1)^{2}-24 F(x 1-1)^{5} F(x 2-1)-12 F(x 1-1)^{5} F(x 2)^{2}\right. \\
& +24 F(x 1-1)^{5} F(x 2)-30 F(x 1-1)^{4} F(x 2-1)^{3}+45 F(x 1-1)^{4} F(x 2-1)^{2} \\
& +30 F(x 1-1)^{4} F(x 2)^{3}-45 F(x 1-1)^{4} F(x 2)^{2}+30 F(x 1-1)^{3} F(x 2-1)^{4} \\
& -40 F(x 1-1)^{3} F(x 2-1)^{3}-30 F(x 1-1)^{3} F(x 2)^{4}+40 F(x 1-1)^{3} F(x 2)^{3} \\
& -12 F(x 1-1)^{2} F(x 2-1)^{5}+15 F(x 1-1)^{2} F(x 2-1)^{4}+12 F(x 1-1)^{2} F(x 2)^{5} \\
& -15 F(x 1-1)^{2} F(x 2)^{4}+12 F(x 2-1)^{5} F(x 1)^{2}-30 F(x 2-1)^{4} F(x 1)^{3} \\
& -15 F(x 2-1)^{4} F(x 1)^{2}+30 F(x 2-1)^{3} F(x 1)^{4}+40 F(x 2-1)^{3} F(x 1)^{3} \\
& -12 F(x 2-1)^{2} F(x 1)^{5}-45 F(x 2-1)^{2} F(x 1)^{4}+24 F(x 2-1) F(x 1)^{5} \\
& +12 F(x 1)^{5} F(x 2)^{2}-24 F(x 1)^{5} F(x 2)-30 F(x 1)^{4} F(x 2)^{3}+45 F(x 1)^{4} F(x 2)^{2} \\
& \left.+30 F(x 1)^{3} F(x 2)^{4}-40 F(x 1)^{3} F(x 2)^{3}-12 F(x 1)^{2} F(x 2)^{5}+15 F(x 1)^{2} F(x 2)^{4}\right)
\end{aligned}
$$

and for integers $x 1$ and $x 2$ with $x 1=x 2$,

$$
\begin{aligned}
& P\left(X_{2: 7}=x 1, X_{6: 7}=x 1\right)=\int_{F(x 1-1)}^{F(x 1)} \int_{F(x 1-1)}^{F(x 1)} f_{2,6}\left(u_{2}, u_{6}\right) d u_{2} d u_{6} \\
& =6 F(x 1-1)^{7}-35 F(x 1-1)^{6}-84 F(x 1-1)^{5} F(x 1)^{2}+168 F(x 1-1)^{5} F(x 1)
\end{aligned}
$$




\section{UCHIDA}

$$
\begin{aligned}
& +210 F(x 1-1)^{4} F(x 1)^{3}-315 F(x 1-1)^{4} F(x 1)^{2}-210 F(x 1-1)^{3} F(x 1)^{4} \\
& +280 F(x 1-1)^{3} F(x 1)^{3}+84 F(x 1-1)^{2} F(x 1)^{5}-105 F(x 1-1)^{2} F(x 1)^{4} \\
& -6 F(x 1)^{7}+7 F(x 1)^{6}
\end{aligned}
$$

Similarly we also obtain the joint pmf of 4 order statistics of a random sample of size 12 with a cdf $F(x)$. For example, we obtain the following equation.

$$
\begin{aligned}
P & \left(X_{1: 12}=x 1, X_{3: 12}=x 2, X_{5: 12}=x 2, X_{9: 12}=x 3\right) \\
= & \int_{F(x 1-1)}^{F(x 1)} \int_{F(x 2-1)}^{F(x 2)} \int_{F(x 2-1)}^{F(x 2)} \int_{F(x 3-1)}^{F(x 3)} f_{1,3,5,9}\left(u_{1}, u_{3}, u_{5}, u_{9}\right) d u_{1} d u_{3} d u_{5} d u_{9} \\
= & 396\left(35 F(x 1-1)^{2} F(x 2-1)^{6} F(x 3-1)^{4}-140 F(x 1-1)^{2} F(x 2-1)^{6} F(x 3-1)^{3}\right. \\
& +210 F(x 1-1)^{2} F(x 2-1)^{6} F(x 3-1)^{2}-140 F(x 1-1)^{2} F(x 2-1)^{6} F(x 3-1) \\
& -35 F(x 1-1)^{2} F(x 2-1)^{6} F(x 3)^{4}+140 F(x 1-1)^{2} F(x 2-1)^{6} F(x 3)^{3} \\
& -210 F(x 1-1)^{2} F(x 2-1)^{6} F(x 3)^{2}+140 F(x 1-1)^{2} F(x 2-1)^{6} F(x 3) \\
& -168 F(x 1-1)^{2} F(x 2-1)^{5} F(x 3-1)^{5}+630 F(x 1-1)^{2} F(x 2-1)^{5} F(x 3-1)^{4} \\
& -840 F(x 1-1)^{2} F(x 2-1)^{5} F(x 3-1)^{3}+420 F(x 1-1)^{2} F(x 2-1)^{5} F(x 3-1)^{2} \\
& -560 F(x 1) F(x 2)^{6} F(x 3)^{5}+2100 F(x 1) F(x 2)^{6} F(x 3)^{4} \\
& -2800 F(x 1) F(x 2)^{6} F(x 3)^{3}+1400 F(x 1) F(x 2)^{6} F(x 3)^{2} \\
& +560 F(x 1) F(x 2)^{5} F(x 3)^{6}-2016 F(x 1) F(x 2)^{5} F(x 3)^{5} \\
& +2520 F(x 1) F(x 2)^{5} F(x 3)^{4}-1120 F(x 1) F(x 2)^{5} F(x 3)^{3} \\
& -200 F(x 1) F(x 2)^{4} F(x 3)^{7}+700 F(x 1) F(x 2)^{4} F(x 3)^{6} \\
& \left.-840 F(x 1) F(x 2)^{4} F(x 3)^{5}+350 F(x 1) F(x 2)^{4} F(x 3)^{4}\right) .
\end{aligned}
$$

\section{Appendix 2.}

Here we obtain the exact distribution of interquartile range of the bootstrap sample. Let $X_{1}, X_{2}, \ldots, X_{7}$ be a random sample of size 7 with a standard normal cdf. Let $X_{1}^{*}, X_{2}^{*}, \ldots, X_{7}^{*}$ be the bootstrap sample of size 7 . When we obtained the standard normal observations that $x_{1}=-0.64408, x_{2}=-0.46, x_{3}=-0.03421, x_{4}=0.290679, x_{5}=$ $0.713922, x_{6}=1.508748, x_{7}=1.692334$, by using Example 4.2 and the REDUCE system, we can obtain the exact distribution of interquartile range $X_{6: 7}^{*}-X_{2: 7}^{*}$ of the bootstrap sample of size 7 , that is, $P^{*}\left(X_{6: 7}^{*}-X_{2: 7}^{*} \leq x\right)$. This distribution is completely determined by the following jump points and their pmf.

$$
\begin{array}{ll}
s_{1}=0.000000, & m(0.000000)=0.0021504646434, \\
s_{2}=0.183586, & m(0.183586)=0.0025159584458, \\
s_{3}=0.184080, & m(0.184080)=0.00251595848675, \\
s_{4}=0.324889, & m(0.324889)=0.0116958070185, \\
s_{5}=0.423243, & m(0.423243)=0.0116958070139, \\
s_{6}=0.425790, & m(0.425790)=0.00863585750748, \\
s_{7}=0.609870, & m(0.609870)=0.0154697447495, \\
s_{8}=0.748132, & m(0.748132)=0.0521891388784,
\end{array}
$$




$$
\begin{array}{ll}
s_{9}=0.750679, & m(0.750679)=0.0430092903447 \\
s_{10}=0.794826, & m(0.794826)=0.00863585757407 \\
s_{11}=0.934759, & m(0.934759)=0.041683312226 \\
s_{12}=0.978412, & m(0.978412)=0.0154697448372 \\
s_{13}=1.173922, & m(1.173922)=0.0998223529273 \\
s_{14}=1.218069, & m(1.218069)=0.0430092903395 \\
s_{15}=1.358002, & m(1.358002)=0.071976812384 \\
s_{16}=1.401655, & m(1.401655)=0.0416833122162 \\
s_{17}=1.542958, & m(1.542958)=0.0998223529303 \\
s_{18}=1.726544, & m(1.726544)=0.0719768123821 \\
s_{19}=1.968748, & m(1.968748)=0.139295701614 \\
s_{20}=2.152334, & m(2.152334)=0.0849305986346 \\
s_{21}=2.152828, & m(2.152828)=0.0849305986476 \\
\text { and } &
\end{array}
$$

\section{Acknowledgements.}

The author would like to thank Dr. Aki of Osaka University for instructive comments and discussions. 
UCHIDA

\section{REFERENCES}

Abdel-Aty (1954). Ordered variables in discontinuous distributions, Statist. Neerland., 8, 61-82.

Arnold, B. C., Balakrishnan, N. and Nagaraja, H. N. (1992). A First Course in Order Statistics, Wiley, New York.

Beran, R. and Ducharme, G. R. (1991). Asymptotic Theory for Bootstrap Methods in Statistics, CRM, Université de Montréal.

Efron, B. (1979). Bootstrap methods : another look at the jackknife, Ann. Statist., 7, 1-26.

David, H. A. (1981). Order Statistics, 2nd ed., Wiley, New York.

Gupta, P. L. and Gupta, R. C. (1981). Probability of ties and Markov property in discrete order statistics, J. Statist. Plann. Inference, 5, 273-279.

Hearn, A. C. (1984). REDUCE User's Manual, Version 3.1, The Rand Corporation, Santa Monica.

Khatri, C. G. (1962). Distributions of order statistics for discrete case, Ann. Inst. Statist. Math., 14, 167-171.

Nagaraja, H. N. (1992). Order Statistics from discrete distributions (with discussion), Statistics, 23(2), 189-216.

Shorack, G. R. and Wellner, J. A. (1986). Empirical Processes with Applications to Statistics, Wiley, New York.

Reiss, R. D. (1989). Approximate Distributions of Order Statistics with Applications to Nonparametric Statistics, Springer, New York.

(Received August 1994; Revised October 1994) 